\title{
HAADF-STEM analysis of the composition distribution in InAlAsSb/InGaAs/InP layers for solar cells applications
}

\author{
N. Baladés ${ }^{1}$, M. Herrera ${ }^{1}$, D. L. Sales ${ }^{1}$, N. Fernández ${ }^{1}$, F.J. Delgado ${ }^{1}$, D. Hernández-Maldonado ${ }^{2}$, Q. $^{3}$ \\ Ramasse $^{2}$, M. González ${ }^{3,4}$, S. Tomasulo ${ }^{3}$, J. Abell $^{3}$, R. Walters ${ }^{3}$ and S. I. Molina ${ }^{1}$. \\ 1. Department of Material Science, Metallurgical Engineering and Inorganic Chemistry, University of \\ Cádiz, Campus Río San Pedro, 11510, Puerto Real, Cádiz, Spain. \\ 2. SuperSTEM. STFC Daresbury Laboratories. Keckwick Lane. Warrington. WA4 4AD. UK. \\ ${ }^{3 .}$ U.S Naval Research Laboratory, 4555 Overlook Ave. SW, Washington D.C. 20375, USA. \\ 4. Sotera Defense Solutions, 430 National Business Pkwy \# 100, Annapolis Junction, MD 20701, USA.
}

The first generation of solar cells were made by simple p-n junctions of crystalline silicon ( $\mathrm{Si}$ ) with efficiencies between 7-8\% [1]. From then on, large research effort in the solar energy field has been aimed at improving their efficiency and reducing the size of the devices. As an example, GaInP/GaAs quantum well tandem cells have been reported to allow the absorption of lower energy photons beyond the GaAs bulk band-gap with efficiencies of 34\% [2] and inverted metamorphic (IMM) triple-junction III-V solar cells on GaAs or Ge substrates have as high as $40 \%$ under concentrated illumination [3], III-V multijunction technology currently holds the record high efficiency of $46 \%$ at 508 Suns [4].

Nowadays, attention is paid to exploring multi-junction solar cells based on ternary and quaternary alloys (InGaAs, InGaAlAs, InGaAsP, InAlAsSb) on InP. In particular, triple junction solar cells with a top junction bandgap energy of $1.75 \mathrm{eV}$ are expected to provide a very high efficiency in the conversion of light [5]. A promising alloy for the top junction in these devices is the quaternary InAlAsSb, which can be grown lattice-matched to InP. The understanding of the structural properties of this material is essential to understand and optimize its optoelectronic properties.

In this communication, we analyse by transmission electron microscopy (TEM) the structural properties of an InAlAsSb/InGaAs/InP heterostructures grown by Molecular Beam Epitaxy (MBE). The photoluminescence emission of the samples grown at different temperatures shows the presence of subbandgap tail of states, suggesting the presence of compositional alloy variations in the quaternary InAlAsSb material. First, we have studied the sample by diffraction contrast in 220 bright field (BF) conditions (Figure 1). Strain contrast associated with composition modulation, due to spinodal decomposition, have been observed in the InGaAs buffer layer. This phase separation can affect the growth of the quaternary layer. In order to characterize possible composition fluctuations in the layers, we have analysed the structure by aberration corrected high angle annular dark field scanning (HAADF-S) TEM, as shown in Figure 2. Strong contrasts variations have not been observed in these images, suggesting that the magnitude of possible composition variations is not large. The simulation of these HAADF-STEM images is in progress to help in the interpretation of the images, in order to shed some light on the composition distribution in these heterostructures. 


\section{References:}

[1] D. A. Kleinman, Bell System Technical Journal 40 (1961) p. 81.

[2] J. G. J. Adams et al., $24^{\text {th }}$ European Photovoltaic Solar Energy Conference (2009) p. 19.

[3] J. F. Geisz et al., Applied Physics Letters 93 (2008) p. 123505.

[4] M. Green, Prog. Photovolt: Res. Appl. 23 (2015) p.1

[5] M. González et al., SPIE Proceedings 7933 (2011) p. 79330R-1.

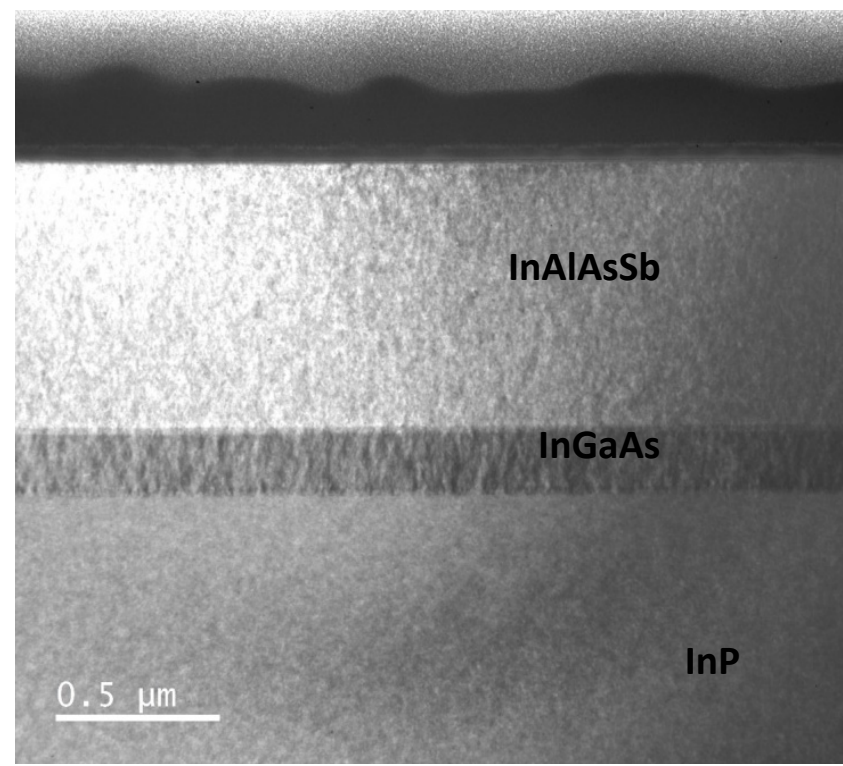

Figure 1. g220BF image of the sample showing strain contrasts associated to spinodal decomposition in the InGaAs buffer layer.

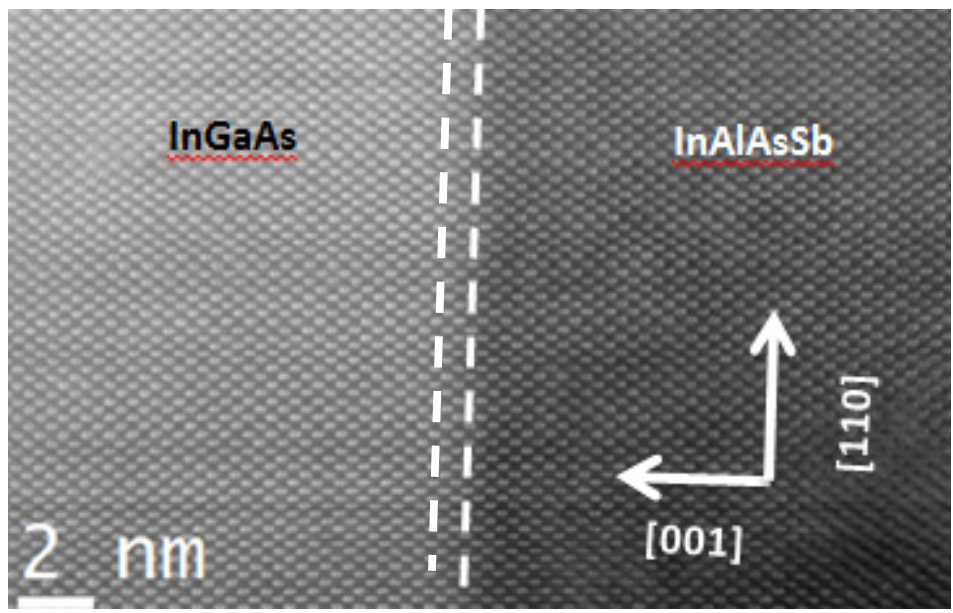

Figure 2. HAADF-STEM image of the interface InAlAsSb-InGaAs, where strong contrast variations have not been found. 